\title{
ÍNDICE DE DESARROLLO SOCIOAMBIENTAL PARA EL EsTADO DE BAHÍA
}

\section{Leonardo Araújo*, Elaine Fernandes** y Patrícia Rosado***}

Fecha de recepción: 10 de abril de 2011. Fecha de aceptación: 1 de septiembre de 2011.

\section{RESUMEN}

Debido a la creciente importancia de las cuestiones ambientales y de la falta de información sobre el tema en el Estado de Bahía, este trabajo analizó cómo las condiciones ambientales influenciaron en el nivel de desarrollo de la población local. Para eso, fue creado el Índice de Desarrollo Socio Ambiental-IDSA- que innova al introducir en el Índice de Desarrollo Humano-IDH-, variables que representan la calidad ambiental de los municipios del Estado. El IDSA mediano obtuvo un valor igual a 0.26 , que está 0.4 puntos abajo del IDH medio. De esta forma, el estudio concluyó que la calidad ambiental se constituye en un importante factor reductor del nivel bahiano de desarrollo humano. Este resultado ocurre, principalmente, como consecuencia de la mala calidad y disponibilidad de los servicios de saneamiento proporcionados por el Estado.

Palabras clave: medio ambiente, indicador, ICA, IDSA, Bahía.

\section{Socio-EnVironmental DeVelopment IndeX FOR THE STATE OF BAHÍA}

\begin{abstract}
This paper analyzes how environmental conditions have influenced the level of development in the local population of the State of Bahía, given the growing importance of environmental issues and the lack of information on these issues in the State. To this end, the Index of Socio-Environmental Development -IDSA- is innovative in introducing the Index of Human Development -IDH-, variables representing the environmental quality of the State municipalities. The IDSA average was 0.26 , a value 0.4 points below that of the IDH. Thus the study concludes that environmental quality is a significant factor in reducing the level of human development in Bahía. This has occurred principally as a result of the poor quality and availability of sanitation services provided by the State.

Key Words: environment, indicator, ICA, IDSA, Bahía

* Economista de la Empresa Brasileńa de Pesquisa Agropecuaria Rondônia. Correo electrónico: araujolv@gmail.com

** Profesora adjunta de la Universidad Federal de Viçosa, Brasil. Correo electrónico: eafernandes@ufv.br

*** Profesora adjunta de la Universidad Federal de São João del-Rei, Brasil. Correo electrónico: patriciarosado@yahoo.com.br
\end{abstract}




\section{INDICE DE DÉVELOPPEMENT SOCIO-ENVIRONNEMENTAL POUR L'ÉTAT DE BAHIA Résumé}

Du fait de l'importance croissante des questions environnementales et du manque d'information sur ce thème pour l'État de Bahia, cet article analyse comment les conditions environnementales ont influé sur le niveau de développement de la population locale. Pour cela, il a été conçu un Indice de Développement SocioEnvironnemental - IDSA (en espagnol) - qui innove en introduisant dans l'Indice de Développement Humain, IDH, des variables représentant la qualité de l'environnement dans les municipalités de cet État. L'IDSA moyen atteint une valeur de 0,26, soit 0,4 points en-dessous de L'IDH moyen. La conclusion de cette étude est que la qualité de l'environnement constitue un facteur qui réduit considérablement le niveau de développement humain de l'État de Bahia. Ce résultat découle, principalement, de la mauvaise qualité et du manque de disponibilité des services d'assainissement fournis par l'État.

Mots clés : environnement, indicateur, ICA, IDSA, Bahia.

\section{ÍNDICE DE DESENVOLVIMENTO SOCIOAMBIENTAL PARA O ESTADO DA BAHIA Resumo}

Devido à crescente importância das questôes ambientais e da falta de informação sobre o tema no Estado da Bahia, este trabalho analisou como as condiçóes ambientais influenciaram no nível de desenvolvimento da população local. Por esse motivo, foi criado o Índice de Desenvolvimento Socioambiental -IDSA - que inova quando incorpora ao Índice de Desenvolvimento Humano, IDH-, variáveis que representam a qualidade ambiental dos municípios do Estado. O IDSA médio calculado foi de 0.26, que está situado 0.4 pontos abaixo do IDH médio. Desta forma, o estudo concluiu que a qualidade ambiental é um fator importante na redução do nível baiano de desenvolvimento humano. Esse resultado ocorre, principalmente, como consequência da má qualidade e disponibilidade dos serviços de saneamento fornecidos pelo Estado. Palavras-chave: meio ambiente, indicador, ICA, IDSA, Bahia.

\section{巴伊亚州社会环境发展指数的制定}

\section{摘要}

由于环境问题日益重要, 且巴伊亚州缺乏该方面的信息, 这项研究分析了 环境条件是如何影响当地发展水平的。为此, 本文创立了社会环境发展指 数（IDSA），这是对人类发展指数(IDH)的借鉴和创新，其中的变量代表 该州诸城市的环境质量。社会环境发展指数均值是 0.26 , 比人类发展指数 的平均水平低 0.4 。因此, 该研究结论认为: 环境质量下降是导致巴伊亚人 类发展水平降低的重要因素。之所以产生这一结果, 主要是因为该州提供 的公共卫生服务质量不高, 且供应不足。

关键词：环境, 指数, 社会环境发展指数, 巴伊亚 


\section{INTRODUCCIÓN}

El desarrollo es visto, según Sen (2008), como la remoción de las trabas que limitan las opciones y las oportunidades de las personas. Los términos libertad, seguridad y garantía de los derechos sociales básicos tienen un papel destacado en su obra. En busca de información sobre el nivel de desarrollo de los países, Sen y Mahbud ul Haq construyeron, en 1990, el Índice de Desarrollo Humano (IDH) hasta hoy utilizado por la ONU para clasificar las naciones como más o menos desarrolladas. Este índice es una medida comparativa del grado de desarrollo de los países y está compuesto por las siguientes variables: expectativa de vida al nacer, educación y PBI per cápita.

El IDH (y sus variantes) $)^{1}$ tiene alcance limitado por no considerar las cuestiones ambientales; es, por tanto, insuficiente para medir el desenvolvimiento de determinada localidad en su versión económica, social y ambiental. Adelante de esta constatación, el presente estudio busca avanzar, en relación a lo que este Índice propone: establecer interrelaciones entre la calidad ambiental y la calidad de vida de la población, por medio de la inclusión de nuevas variables (principalmente ambientales) a la composición del IDH. Se debe resaltar que entre estas nuevas variables, las ambientales son consideradas extremadamente importantes para determinar el Índice de Desenvolvimiento Humano, pues según Sachs (2007), afectan y son afectadas por la calidad de vida de la población.

El Estado de Bahía fue elegido como objeto de análisis puesto que posee características peculiares en términos fisiográficos, de localización e infra-estructurales, con disponibilidad de recursos naturales y de materias primas. Además, posee una gran concentración de población y de sus actividades económicas sobre el mismo espacio (la región costera del Estado), que causó presiones sobre el medio ambiente y consecuentes alteraciones de las condiciones ambientales de los municipios ahí localizados. Poca importancia se da a la influencia de esta cuestión sobre la calidad de vida de la población. Según Ross, mono culturas extensivas han revelado ser un importante factor de degradación ambiental, pues además de la erosión de los suelos, contribuyen a la sedimentación de los cursos de agua y deterioran la calidad de las aguas de arroyos y ríos (Ross, 2001). Estas presiones sobre el medio ambiente generan alteraciones negativas de la calidad

1 Llamados índices de tercera generación. Como el ICv (Índice de Condición de Vida), construido para los municipios mineros por la Fundación João Pinheiro; el IPRs (Índice Paulista de Responsabilidad Social), construido para los municipios paulistas por la Fundación Seade; el IDG (Índice de Desenvolvimiento ajustado al Género); el IPH (Índice de Pobreza Humana), también desarrollados por el Programa de las Naciones Unidas para el Desarrollo (PNUD) (Braga et al., 2003). 
ambiental y de la calidad de vida de la población en virtud del modelo de desenvolvimiento adoptado, basado en la explotación de los recursos naturales con una tasa superior a la capacidad de regeneración natural y en la gestión de las materias primas y de los residuos de manera inadecuada (SEPLAN, 2009).

En este contexto el presente artículo analiza de forma general, las condiciones socioeconómicas y ambientales para el Estado de Bahía, busca evidenciar cómo el fenómeno de la calidad ambiental se asocia a la calidad de vida de la población. De manera específica se pretende: $\boldsymbol{a}$ ) verificar cuáles son los indicadores que mejor explican la calidad ambiental; $\boldsymbol{b}$ ) construir Índices de Condiciones Ambientales para los municipios de Bahía (ICA); y c) construir Índices de Desenvolvimiento Humano Ambiental para los municipios de Bahía.

Ha habido algunos intentos en bibliografía especializada en construcción de índices, con diferentes grados y formas de agregación que incorporan cuestiones ambientales. Sin embargo, de acuerdo con Hales y Prescott-Allen (2005), en la práctica, los únicos Índices de Sustentabilidad que adquirirán gran visibilidad a nivel internacional son aquéllos divulgados por World Wide Fund for Nature (WwF) y World Economic Forum (WEF), calculados por dos de las más importantes instituciones académicas del área, el Yale Center for Environmental Law and Policy, y el Center for International Earth Science Information Network, de la Universidad de Columbia. ${ }^{2}$

Incluso con mayor visibilidad, estos índices son limitados pues no tienen en cuenta las peculiaridades y diferentes características de los países en su construcción. Por lo tanto, este estudio intenta llenar la brecha existente entre la necesidad de identificar los problemas relevantes relacionados al medio socio ambiental y las posibles alternativas que posibilitan su desenvolvimiento de forma sustentable y más equitativa.

Este artículo está organizado en cuatro secciones, además de esta introducción. En la próxima, se contextualiza la interrelación entre calidad de vida y calidad ambiental. En la segunda se hace un resumen del método de análisis. En la tercera, se discuten los resultados. En la cuarta sección se presentan algunas consideraciones finales.

\section{CONTEXTUALIZANDO CALIDAD DE VIDA Y CALIDAD AMBIENTAL}

Según Sen (2008), las estrategias de desarrollo de los países tienen que contemplar acciones en el sentido de crear un clima económico, social, político y

2 Para mayores detalles ver Prescott-Allen (2005). 
cultural favorable a sus individuos. Ello porque el desempeño de cada persona depende de las oportunidades económicas, de las libertades políticas, cuestiones sociales y educacionales, y del estímulo de sus iniciativas. De esta manera, la calidad de vida del individuo está íntimamente relacionada con las oportunidades efectivas dadas por las realizaciones colectivas, tanto pasadas como presentes.

Sachs (2007) complementa a Sen, y dice que el desarrollo sustentable debe enfatizar, además de la problemática económica y social, en cuestiones ambientales. Esto significa, por ejemplo, que la elevación de calidad de vida y la equidad social deben ser objetivos centrales (propósito final) del modelo de desarrollo. De la misma manera, la eficiencia y el crecimiento económico son esenciales, pues se hace improbable aumentar la calidad de vida con equidad sin que la economía tenga condiciones de crecer económicamente. La preservación ambiental o prudencia ecológica es otro hecho extremadamente importante. En la ausencia de condiciones ambientales adecuadas, no es posible asegurar la calidad de vida y la equidad social a las generaciones futuras.

Gallopín (1982) completa enfatizando que las condiciones ambientales se relacionan íntimamente a la calidad de vida, pues ésta es vista como resultante de la salud de la persona y de su sentimiento de satisfacción.

Con el objetivo de relacionar variables representativas de la calidad de vida y de la calidad ambiental citadas anteriormente, Bojö et al. (2001) construyen la representación esquemática ilustrada en la Gráfica 1. La Gráfica muestra los efectos de calidad ambiental (tercera línea de arriba para abajo) sobre la calidad de vida (rectángulos), y los efectos de ambas sobre el bienestar.

Gráfica 1. Representación esquemática de las dimensiones de la calidad ambiental y de vida.
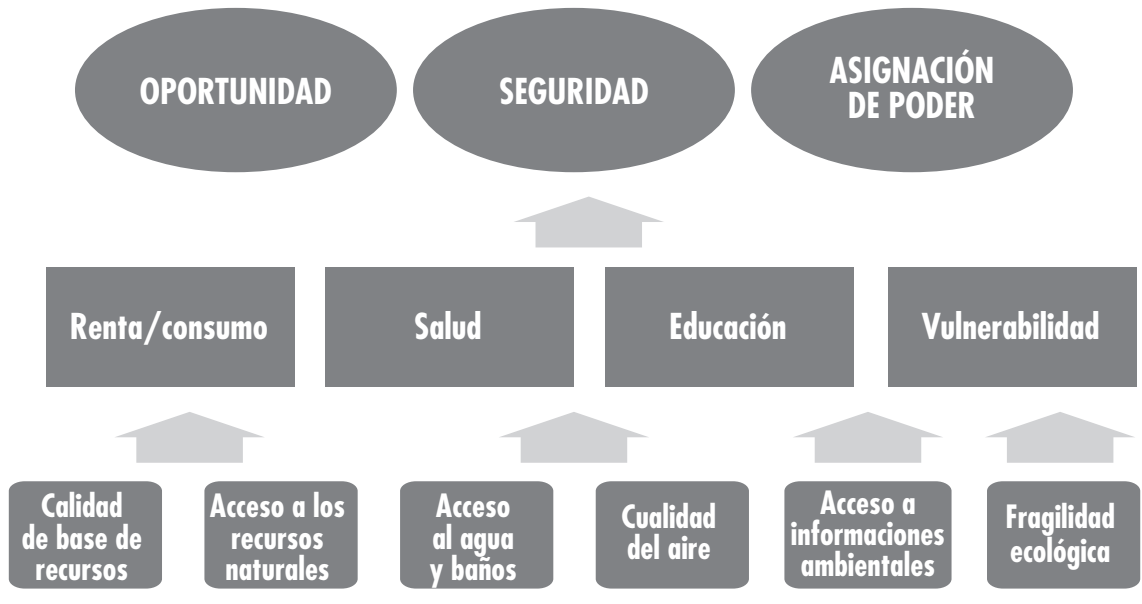

Origen: Adaptado de Bojö et al. (2001). 
El ser humano hace parte del medio ambiente con todo lo que produce artificialmente, afecta y es afectado por él. La calidad de vida y la calidad ambiental están directamente relacionadas, pues la polución del aire, de las aguas y del suelo, además de la extinción de ecosistemas, afecta directamente el bienestar de los individuos. Se puede entonces, llamar calidad ambiental al estado en que se encuentran las varias dimensiones del medio ambiente. Si el ambiente, que abarca la humanidad con sus prácticas y costumbres, es dañado de alguna forma, hay una reducción del nivel de calidad de vida. La calidad de vida afecta la calidad ambiental porque es un componente de ésta (Sachs, 2007).

La calidad de vida resulta de la exposición de los individuos a circunstancias adversas, guardando relación directa con factores tales como el medio ambiente saludable y productivo, la existencia de espacios estéticos y recreativos y la participación de los individuos en la toma de decisiones. Ese concepto está relacionado todavía con oportunidades de satisfacción de los deseos y aspiraciones humanas. La calidad de vida va más allá del suministro de las necesidades básicas del individuo (alimentación, salud, habitación, vestuario, educación, empleo y participación) en función de los valores culturales (Leff, 2004). De ese modo, es imposible comprender que si la calidad ambiental es un componente de la calidad de vida y ésta, a su vez, es probablemente el componente más importante del desarrollo, se puede concluir que el desarrollo depende de una buena gestión del medio ambiente (Sachs, 2007). En este sentido, el impacto causado al medio ambiente por los seres humanos y el impacto del medio ambiente sobre la calidad de vida de éstos es cada vez más complejo, tanto en términos cuantitativos como cualitativos (Leitte, 2000).

Y la calidad de vida depende de la calidad ambiental, desde que se quiere alcanzar el desarrollo equilibrado y sustentable. ${ }^{3}$ Esa idea de calidad de vida tiene como meta romper los parámetros homogéneos del bienestar y crear oportunidades para la formación de nuevos índices que sean capaces de articular los costos del crecimiento con los valores culturales y el potencial de la naturaleza, además de complementar las medidas objetivas con las percepciones subjetivas.

3 Con todos estos acontecimientos, la CMMAD trabajó entre 1984 y 1987 en la creación de una agenda que posibilitara un cambio global; el resultado fue la publicación del relato Our Common Future (Relato Brundtland), en el cual se examinaban los problemas críticos entre desarrollo y medio ambiente. Aquí es conceptualizado el término desarrollo sustentable, el cual tendría que responder a las necesidades del presente de forma equitativa, sin comprometer las posibilidades de sobrevivencia y prosperidad de las generaciones futuras (Brundtland, 1987). 
Es así que la calidad de vida abre perspectivas para que se pueda reflexionar acerca de equidad social, junto a las esferas de la diversidad ecológica y cultural (Leff, 2004).

De forma sintética, el significado de calidad de vida conduce a los valores que reglamentan el comportamiento social. Esos valores están asociados, no solamente al nivel de renta o a la distribución de la riqueza, sino también a criterios de salud reproductiva, calidad de la maternidad y de la paternidad y, finalmente, a los mecanismos de autogestión ambiental. Por tanto, el deterioro de la calidad ambiental generalmente da origen al estado patológico de reincidencia de las enfermedades de la pobreza ${ }^{4}$ (como el cólera y el dengue), que provienen de la contaminación del aire, de los suelos, de los recursos hídricos y del uso de recursos tóxicos como pesticidas.

Ante el agravamiento de los problemas ambientales, la acción política debe privilegiar métodos que dinamicen el acceso a la conciencia ambiental de la población por intermedio de la educación. Además, la comprensión de los problemas ambientales debe estar basada en la dimensión socio ambiental, considerando especialmente los criterios culturales que, a su vez, exigirán determinaciones específicas en la formulación de políticas públicas. El gran desafío es perseguir las metas de calidad de vida y calidad ambiental de modo que se puedan reforzar mutuamente. ${ }^{5}$

\section{METODOLOGÍA Área de Estudio}

El Estado de Bahía, con 415 municipios que ocupan un área de 567.692,669 $\mathrm{km}^{2}$, está localizado en la región Nordeste de Brasil, y posee un total de ocho estados limítrofes. De acuerdo con datos del año 2000, Bahía es el cuarto estado brasileño más habitado, con una población de 13.070.250 habitantes y una densidad demográfica media de $54 \mathrm{hab} / \mathrm{km}^{2}$. A Bahía le corresponde el 36\% del PBI del Nordeste y más de la mitad de las exportaciones (IBGE, 2009).

4 Estudios como los de Ekbom y Bojö (1999), Barbier (2000) y Parikh (2002) muestran la relación entre pobreza y medio ambiente.

5 Se puede encontrar en bibliografía nacional e internacional algunos trabajos que tratan sobre la construcción de índices que incorporan las condiciones ambientales. Para mayores detalles consultar Veiga (2008). 


\section{Fuente de datos}

Fueron utilizados en esta investigación datos referentes a todos los municipios del Estado de Bahía en el año 2000. Se extrajeron las siguientes variables del Censo Demográfico con data-base 2000: personas que viven en domicilios con servicio de recolección de basura (X1); personas que viven en domicilios con agua corriente $(\mathrm{X} 2)$; personas que viven en domicilios con red general de alcantarilla o pluvial (X3); personas que viven en domicilios con iluminación eléctrica (X4); porcentaje de personas que viven en domicilios sin automóvil (X5) (para esta última fue hecha la transformación de la variable "porcentaje de personas que viven en domicilios con automóvil" restando la misma en 100); renta per cápita (X6); esperanza de vida al nacer (X7); frecuencia a la escuela (X8).

La variable participación del sector industrial en el PBI municipal (X9), del año 2002, fue obtenida junto a la Superintendência de Estudos Econômicos e Sociais do Estado da Bahia (SEI, 2009). Por último, se obtuvieron en el Censo Agropecuario del IBGE (2006), informaciones referentes a áreas de matas y florestas que cubrían los municipios. Este valor fue dividido por el área municipal para obtener el porcentaje del área de los municipios cubiertos por vegetación nativa (X10).

\section{Construyendo el Índice de Condiciones Ambientales de los municipios de Bahía}

El Índice de Condiciones Ambientales (ICA) es utilizado como medida de la proporción de la calidad ambiental del área de determinado municipio. Su construcción fue hecha en dos etapas. En la primera, se desarrolló el Índice Parcial de las Condiciones Ambientales (IPCA) por medio del análisis factorial multivariado. En la segunda, con base en el IPCA, fueron estimados los pesos atribuidos a cada una de las variables que entraron en la composición del ICA, utilizando el análisis de regresión con aplicación del método de los mínimos cuadrados restrictos (MQR).

Los factores estimados por la técnica deben explicar parte significativa de la variación del conjunto de variables originales, teniendo en cuenta que el primer factor contiene el más grande porcentaje de explicación de la variación total, el segundo factor tiene el segundo porcentaje más grande, y así sucesivamente (Corrar et al., 2007).

La propiedad de ortogonalidad de la puntuación de los factores estimados fue utilizada para la elaboración del índice parcial. Entre tanto, se debe observar que la ortogonalidad, asociada a la matriz de factores, no implica necesariamente la ortogonalidad de la puntuación de los factores, debiéndose evaluar si los 
puntajes factoriales son ortogonales por medio de la matriz de variación y covarianza entre estos puntajes (Corrar et al., 2007).

El IPCA puede ser estimado por medio de la expresión:

$$
\text { IPCA } \quad=\left(\sum_{i=1}^{n} F_{i j}^{2}\right)^{\frac{1}{2}} \text { com } \quad j=1,2, \cdots p
$$

en que IPCA $\mathrm{I}_{\mathrm{i}}$ es el Índice Parcial de Condiciones Ambientales asociado al i-eximo municipio de Bahía, y $\mathrm{F}_{\mathrm{ij}}$ son los puntajes factoriales estimados, conforme el procedimiento de los componentes principales. El análisis factorial fue conducido por indicadores presentados en la fuente de datos, con excepción de las variables, renta per cápita; esperanza de vida al nacer y frecuencia a la escuela que fueron utilizadas para la construcción del Índice de Desarrollo Socioeconómico Ambiental (IDSA). De este modo, se utilizaron los siguientes indicadores: (X1) personas que viven en domicilios con recolección de basura; (X2) personas que viven en domicilios con agua corriente; (X3) personas que viven en domicilios que poseen red general de alcantarilla o pluvial; (X5) porcentaje de personas que viven en domicilios sin automóvil; y (X9) participación de la industria en el PBI del municipio. Esas variables representan la infraestructura del Estado y son utilizadas en el presente análisis como una proxy de calidad ambiental y, por tanto, de calidad de vida. La última variable analizada $(\mathrm{X} 10=$ cobertura vegetal $)$, representa directamente la calidad ambiental y fue definida como el valor de las áreas de matas y florestas de los municipios dividido entre el valor del área total municipal.

El Índice Parcial refleja sólo un ranking de los municipios en lo que se refiere a condiciones ambientales. De este modo, no sirve para estimar el porcentaje de calidad ambiental de cada uno de los municipios. Para eso se ha construido el ICA. Ese índice fue construido a partir de la incorporación de pesos a cada una de las variables utilizadas en la composición del Índice Parcial obtenido anteriormente (Ecuación 1). Estos pesos fueron obtenidos a través del análisis de regresión por el método de los minimos quadrados restritos (mínimos quadrados restrictos) (MQR) en que la variable IPCA es variable dependiente y los indicadores (X1), (X2), (X3), (X4), (X5) y (X8) son las variables explicativas. Las Ecuaciones (2) y (3) muestran cómo está hecho el ICA.

$$
\begin{aligned}
& I P C A=\beta_{1} X 1+\beta_{6} X 2+\beta_{2} X 3+\beta_{3} X 4+\beta_{5} X 5+\beta_{7} X 9+\beta_{8} X 10 \\
& I C A=\left(\sum_{i=1}^{n} P_{j} X_{i}\right)
\end{aligned}
$$

en que los pesos $\mathrm{P}_{\mathrm{j}}$ son los parámetros estimados por la Ecuación (3) con sumatorio igual a $1 ; \mathrm{X}_{\mathrm{i}}$ son variables utilizadas para la construcción del IPCA. 


\section{ÍNDICES DE DESARROLLO SOCIOECONÓMICO AMBIENTAL (IDSA)}

Después de la construcción del Índice de Condiciones Ambientales (ICA), se buscó construir un índice que reflejara el nivel de desarrollo humano de los municipios bahianos utilizando también cuestiones relacionadas al medio ambiente. Se trata del Índice de Desarrollo Socioeconómico Ambiental, construido con el objetivo principal de describir las condiciones de vida de los municipios del Estado de Bahía considerando, además de las informaciones relativas a las condiciones económicas, al nivel de instrucción y a la longevidad, las informaciones de naturaleza ambiental de los municipios bahianos.

Para la construcción del IDSA fueron utilizados los procedimientos metodológicos desarrollados en la sección anterior para la construcción de Índices de Condiciones Ambientales, pero adaptados para el caso específico. Fue necesario, inicialmente, la estimativa y la construcción de un índice parcial, así como se hizo en la construcción del ICA. Ese índice es llamado Índice Parcial de Desarrollo Socioeconómico Ambiental (IPDSA). Estimado y construido por medio de la Ecuación 4, siendo los factores comunes obtenidos también a través del empleo de la técnica de análisis factorial por componente principal.

$$
\operatorname{IPDSA}_{i}=\left(\sum_{i=1}^{n} F_{i j}^{2}\right)^{\frac{1}{2}} \operatorname{com} \quad j=1,2, \cdots p
$$

en que el IPDSA es el Índice Parcial de Desarrollo Socioeconómico Ambiental asociado al i-eximo municipio en cuestión; $\mathrm{F}_{\mathrm{ij}}$ son los escores factoriales estimados según el procedimiento de descomposición de componentes principales. El análisis factorial fue conducido para los siguientes indicadores: Esperanza de Vida al nacer (EV), Renta Per Cápita (RPC), Tasa Bruta de Frecuencia a la Escuela (TXESC) e Índice de Condiciones Ambientales (ICA).

Después de la construcción del Índice Parcial de Desarrollo Socioeconómico Ambiental, fueron asociados pesos, por medio del MQR, a cada una de las dimensiones supra citadas. Ese índice fue utilizado como variable dependiente y la Renta Per Cápita, Esperanza de Vida al nacer, Tasa de Frecuencia Escolar y el Índice de Condiciones Ambientales fueron las variables explicativas. Fue hecha la construcción del IDSA $A_{\mathrm{i}}$ asociado al i-eximo municipio de la siguiente forma:

$$
\text { IDSA }=\left(\sum_{i=1}^{n} P_{j} X_{i}\right)
$$

en que los pesos $\mathrm{P}_{\mathrm{j}}$ son estimados por regresión múltiple y $\mathrm{X}_{\mathrm{i}}$ son las variables explicativas. 
Para mejor visualización y entendimiento de los resultados del estudio, se ha adoptado en este trabajo la misma clasificación que la onU utiliza para distribuir el IDH. El mismo es clasificado en cuatro grupos: desarrollo humano muy elevado (con un IDH de 0,900 o superior), desarrollo humano elevado mediano (IDH de 0,800 a 0,899 ), desarrollo humano mediano (IDH de 0,500 a 0,799 ) y desarrollo humano bajo (IDH abajo de 0,500) (UNDP, 2009).

\section{RESULTADOS Y DISCUSIONES Índice de las Condiciones Ambientales de los municipios de Bahía}

Teniendo en cuenta el objetivo de construir un índice de medida del nivel de desarrollo de los municipios bahianos y considerando las condiciones ambientales, el presente trabajo elaboró inicialmente el ICA para cada municipio del Estado de Bahía. El índice fue estimado para ser incorporado al IDH tradicional y así tornarlo más representativo de la calidad de vida real de la población.

Los tests indicaron que la muestra se presentó adecuada al análisis factorial. ${ }^{6}$ Este análisis fue realizado por el método de componentes principales, presentando dos factores con raíces características mayores que uno, conforme pueden ser observadas en el Cuadro 1. Estos resultados muestran que los Factores 1 y 2 contribuyen con $61.07 \%$ para explicar la variación total de los indicadores utilizados. Elegimos la rotación ortogonal de los datos a través del método Varimax para mejor interpretación de los datos.

Cuadro 1. Auto valores de la matriz de correlación y variación explicada por cada uno de los factores en la construcción del ICA del Estado de Bahía

\begin{tabular}{lccc}
\hline Factor & Auto valores & Variancia Explicada por el Factor (\%) & Variancia Acumulada (\%) \\
\hline F1 & 2,367 & 39,458 & 39,458 \\
F2 & 1,297 & 21,619 & 61,077 \\
\hline
\end{tabular}

Origen: Resultados de la investigación.

$6 \quad$ Fue hecho el test de esfericidad de Bartlett y el test de Kaiser-Meyer-Olkin. El test de Bartlett atingió valor igual a 673,843 , significativo a $1 \%$ de probabilidad, lo que permite rechazar la hipótesis nula de que matriz de correlación es una matriz identidad, es decir, no existe correlación entre las variables. Para el test de Kaiser-Meyer-Olkin (кмо), el valor obtenido fue 0,747, indicando que la muestra es adecuada a la realización del análisis factorial. 
Por medio del Cuadro 2 se puede verificar cuáles factores están relacionados a cuáles variables al exhibir las cargas factoriales y comunes. El Factor 1 está fuertemente correlacionado con las variables, personas que viven en domicilios particulares permanentes con agua corriente; personas que viven en domicilios particulares permanentes con servicio de recolección y alcantarilla; personas que viven en domicilios particulares permanentes con servicio de recolección de basura y la participación del sector industrial en el рві municipal. Como fue mencionado anteriormente, la participación relativa del PBI industrial en el PBI municipal fue utilizada como proxy de la contaminación industrial; sin embargo la carga factorial encontrada de 0,442 es inferior a 0.65 en valores absolutos, lo que clasifica esta variable como no relevante para la formación del ICA. El Factor 2 está fuertemente correlacionado con la cobertura vegetal del municipio y personas que viven en domicilios sin automóviles. En relación a este último ítem, se debe considerar que el automóvil es un importante contaminador del aire, principalmente en razón de la emisión de monóxido de carbono.

Cuadro 2. Cargas factoriales y comunes de las variables que forman el Índice de Condiciones Ambientales de los municipios del Estado da Bahía

\begin{tabular}{lccc}
\hline \multirow{2}{*}{ Variables } & \multicolumn{2}{c}{ Cargas Factoriales } & \multirow{2}{*}{ Comunes } \\
\cline { 2 - 2 } & Factor 7 & Factor 2 & \\
\hline Domicilios con abastecimiento de agua & 0,800 & & 0,725 \\
Domicilios con servicio de alcantarilla sanitaria & 0,875 & & 0,768 \\
Domicilios con recolección de basura & 0,863 & & 0,786 \\
Cobertura vegetal & & 0,535 & 0,302 \\
Domicilios sin automóviles & & 0,863 & 0,749 \\
Participación de la industria en el PBI municipal & 0,442 & & 0,335 \\
\hline
\end{tabular}

Origen: Resultados de la investigación.

Se puede decir, entonces, que el Factor 1 sintetiza las variables que captan la infraestructura de los domicilios, principalmente en relación con las condiciones de saneamiento (como el agua corriente, colecta de alcantarilla, tratamiento dado a la basura). Estas variables están fuertemente relacionadas a las condiciones de habitación, o sea, cuanto mejor es la condición de infraestructura de los domicilios, mejor la calidad ambiental.

El Factor 2 resume las variables relacionadas a la calidad ambiental del suelo, del agua y del aire, como cobertura vegetal y porcentual de personas que viven en domicilios sin automóviles. Cuanto mayor es la cobertura vegetal, mayor es el impacto positivo sobre la fijación del suelo, alimentación de los manantiales 
de agua, secuestro de carbono del aire y liberación del oxígeno por la fotosíntesis; además de eso, cuanto mayor es el número de domicilios sin automóvil mejor será la calidad del aire y, consecuentemente, la calidad ambiental.

Después de obtener los factores y cargas factoriales, fueron estimados los puntajes factoriales y calculado el Índice de Condiciones Ambientales.

El ICA para el Estado de Bahía muestra un valor mediano de 0.30, lo que significa que, en media, apenas $30 \%$ de las condiciones ambientales ideales fueron alcanzadas por los municipios bahianos. Esta media está abajo del esperado y refleja, dentro de otros factores, las malas condiciones dadas al destino de la basura doméstica y a la infraestructura de las residencias (acceso al agua y acceso a la alcantarilla sanitaria). En suma, se observa (Gráfica 2) que de 333 municipios bahianos, 80.24\% presentaron un Índice de Condiciones Ambientales igual o inferior a 0.49, considerado bajo. Eso significa que más de tres cuartos de los municipios bahianos están situados en un nivel ambiental que compromete el bienestar de la población.

Se debe destacar que los municipios con peores condiciones ambientales son, respectivamente: Itaguaçu de Bahía, Souto Soares, Ibitiara, Rio do Antônio, Planaltino, Pé de Serra, Ibiassucê, Gentio do Ouro, Caturama y Érico Cardoso. Estos municipios presentan, en media, un área de cobertura vegetal y domicilios sin automóviles inferior a la media de todo el estado, el que reflejó negativamente en la calidad del aire. La cobertura vegetal, además de ejercer funciones ecológicas en el sentido de mejoría del ambiente urbano, proporciona beneficios como la absorción de la polución atmosférica. De este modo, el hecho de que estos municipios no posean cobertura vegetal adecuada y posean muchos autos en sus domicilios contribuye para este resultado.

En el otro extremo, con el ICA más elevado están los municipios de Madre de Deus e Itapetinga. Éstos fueron los únicos que alcanzaron índice superior a 0.8 , considerado como elevado (Gráfica 2). En estos municipios, a pesar de ser relativamente alta la participación del sector industrial (38.3\% en la media) en relación a la media del estado (12.4\%), los demás indicadores utilizados para cuantificar las condiciones del medio ambiente presentaron valores muy superiores de la media estadual. El acceso al agua, alcantarilla sanitaria y servicio de recolección de basura pueden ser citados como ejemplos.

Se observa que la situación de saneamiento básico en el estado es bastante problemática. De entre los municipios clasificados como de bajo ICA, menos de $1 \%$ de la población de éstos tenía acceso al servicio de alcantarilla sanitaria. Cuando se considera todo el estado, sólo $48 \%$ de los municipios bahianos poseen más de 50\% de sus domicilios con acceso al agua corriente. En suma, cuando se refiere al servicio de alcantarilla sanitaria, más de $72 \%$ de los municipios presenta menos de $25 \%$ de sus domicilios con acceso al servicio (Cuadro 3 ). 
Gráfica 2. Índice de Condiciones Ambientales de los municipios bahianos

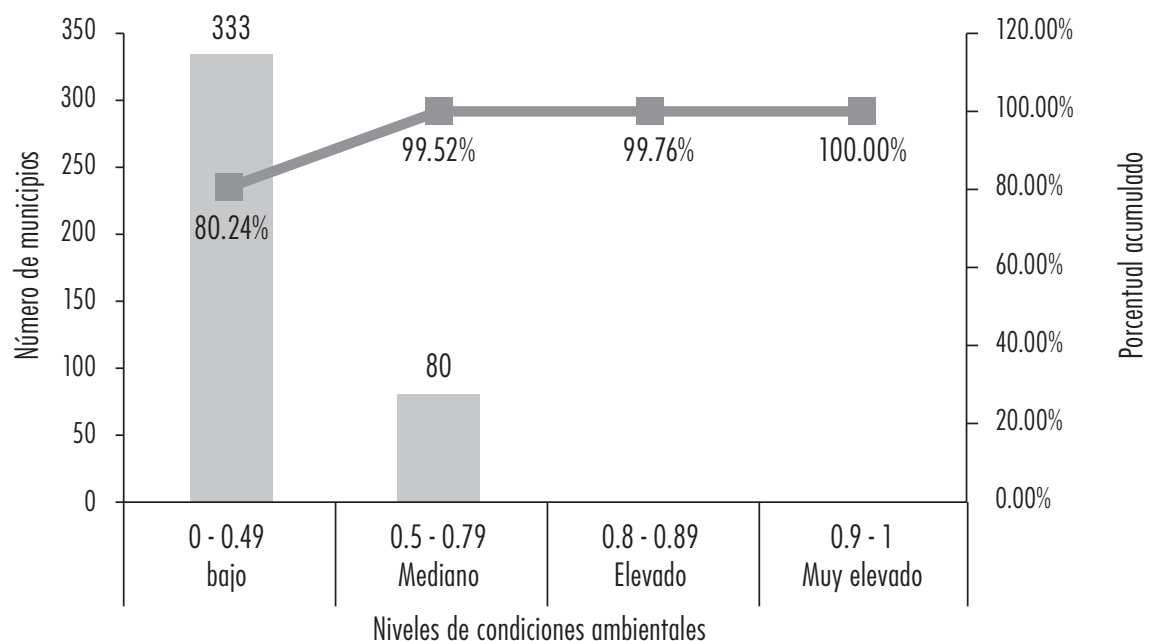

Origen: Resultados de la investigación.

Cuadro 3. Frecuencia de las fajas de acceso al agua y alcantarilla sanitaria en los municipios bahianos, 2000

\begin{tabular}{lcc}
\hline Fajas de cobertura de los domicilios & Con acceso al agua corriente & Con acceso a alcantarilla sanitaria \\
\hline Entre 0\% y 24.9\% & 42 & 302 \\
Entre 25\% y 49.9\% & 213 & 75 \\
Entre 50\% y 74.9\% & 143 & 35 \\
Entre 80\% y 100\% & 16 & 2 \\
\hline
\end{tabular}

Origen: Resultados de la investigación.

En la Gráfica 3 se observa que los municipios que presentan los Índices de Condiciones Ambientales abajo de 0.49 (considerado de baja calidad) están en su mayoría localizados en el interior del estado. Se puede inferir que eso ocurre debido a la baja tasa de urbanización, y consecuentemente un bajo nivel de universalización de los servicios básicos de saneamiento. En suma, la extensión de matas en estas regiones es reducida, pues se trata de área de caatinga (desierto tropical en el nordeste de Brasil, NE) y cerrado donde la densidad forestal es 
naturalmente inferior, además de que viene ocurriendo una gran expansión de actividad agrícola en la región en las últimas dos décadas.

Se puede observar todavía que la microrregión de Ilhéus-Itabuna presenta un aglomerado de municipios con ICA superior a 0.5. Es importante considerar que esta microrregión tiene una mono cultura de cacao de gran importancia económica, y los cacahueros hacen uso muchas veces de las sombras provenientes de la vegetación nativa de la Mata Atlántica. Además de eso, esta microrregión posee significativa cantidad de parques estaduales y reservas indígenas y es una región costera con nivel de urbanización menor que la Región Metropolitana de Salvador.

En la Región Metropolitana de Salvador (RMS), la alta densidad demográfica implicó mayor presión de las actividades humanas sobre el medio ambiente, reduciendo la cobertura vegetal que interfiere en la calidad ambiental de los espacios urbanos, así como en la calidad de vida. Problemas urbanos como de la erosión, sedimentación de los cursos de agua, constitución de islas de calor, falta de áreas verdes, contaminación del aire, sonora y del agua, uso de áreas para deposición de basura, son problemas resultantes de la falta de concientización de parte de la población e inadecuado planeamiento o la falta de éste. Es una microrregión con una renta per cápita relativamente alta, que da como resultado también una flota mayor de vehículos, y un deterioro mayor de la calidad del aire.

Gráfica 3. ICA en el Estado de Bahía según clasificación de la ONU para el IDH. 2000

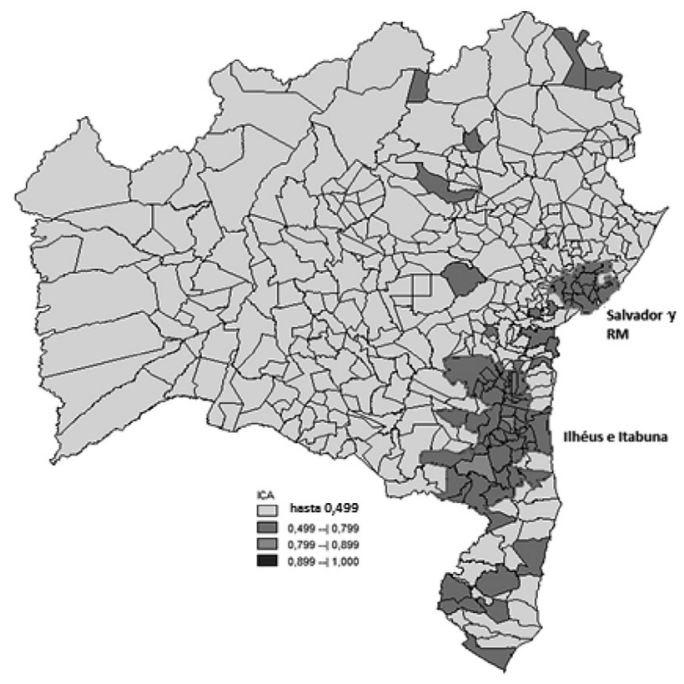

Origen: Resultados de la investigación. 
A pesar de esta presión poblacional, la región presentó un índice de ICA superior a 0.5 . Eso es posible porque los servicios de infraestructura urbana como recolección de basura, abastecimiento de agua y servicio de alcantarilla sanitaria presentan ciertas universalizaciones en esta región.

En suma, es importante observar que hubo un crecimiento considerable de las áreas de la agricultura y pastizales plantadas en relación a las áreas naturales en Brasil y, especialmente, en Bahía. Este resultado tiene serias implicaciones sobre la sustentabilidad, pues en este contexto ocurre un aumento en el uso de fertilizantes y agrotóxicos, y también en el riesgo de contaminación ambiental. Además de eso, la expansión de la agricultura como soja y maíz, por ejemplo, acarrea presiones sobre la agricultura familiar, importante generador de empleo y renta. En 2008 el maíz representó 40.5\% de la producción nacional de cereales, leguminosas y oleaginosas, posicionándose después la soja, principal producto agrícola nacional. Los municipios de Sorriso, en Mato Grosso, y São Desiderio, en Bahía, se destacaron con mayores áreas plantadas de estos granos, 847.886 y 476.422 hectáreas respectivamente. Se observa todavía que el municipio de São Desiderio también se destaca en la producción de algodón herbáceo; inclusive es el mayor productor nacional de este tipo de algodón (IBGE, 2010).

\section{ÍNDICE DE DESARROLLO SOCIOECONÓMICO AMBIENTAL PARA LOS MUNICIPIOS DE BAHÍA}

La elaboración del IDSA incluye los valores estimados para el ICA. Eso fue hecho también por medio de métodos de análisis factorial por componente princi$\mathrm{pal}^{7}$ para los indicadores de Tasa Bruta de Frecuencia Escolar; Renta Per Cápita; Esperanza de Vida al nacer e ICA.

El análisis realizado resultó en dos factores que poseyeron un poder de explicación de la variancia total de los datos del orden de $66 \%$.

De acuerdo con el Cuadro 4, el Factor 1 (F1) está directamente relacionado al ICA, Renta Per Cápita y la Tasa Bruta de Frecuencia Escolar. Eso demuestra

7 Fueron hechos los tests de Bartletty KMO. El test de Bartlett, que para este modelo alcanzó un valor igual a 139,906, fue significativo a $1 \%$ de probabilidad, siendo posible rechazar la hipótesis nula de que no existe correlación entre las variables. Para el test de Kaiser-Meyer-Olkin (кмо), el valor obtenido fue 0.626 , que indica que la muestra se adecua a la realización de análisis factorial. 
justamente la relación anteriormente abordada de que localidades de mayor poder adquisitivo ejercen una mayor presión en el medio ambiente, reflejado consecuentemente en un bajo ICA.

El Factor 2 tiene una relación directa y fuerte con el indicador Esperanza de Vida al nacer: indicador de las condiciones de salud en general. Las similitudes de este factor muestran que $88 \%$ de las variaciones de este indicador es explicada por el Factor 2.

Cuadro 4. Cargas factoriales y similitudes

\begin{tabular}{lccc}
\hline \multirow{2}{*}{ Variables } & \multicolumn{2}{c}{ Cargas Factoriales } & \multirow{2}{*}{ Similitudes } \\
\cline { 2 - 3 } & Factor 1 & Factor 2 & \\
\hline ICA & 0,791 & 0,668 \\
Esperanza de vida al nacer & & 0,966 & 0,880 \\
Renta per cápita & 0,671 & & 0,617 \\
Tasa bruta de frecuencia escolar & 0,688 & 0,391 \\
\hline
\end{tabular}

Origen: Resultados de la investigación.

Los municipios que presentaron los más elevados IDSA fueron Salvador, Lauro de Freitas, Itabuna, Madre de Deus y Paulo Afonso. En el Cuadro 5 son presentados los valores de los $\beta$ referentes a la Ecuación 2, para cada una de las variables. Se observa que los indicadores de mayor peso en este cálculo del IDSA son justamente los indicadores socioeconómicos (esperanza de vida y renta per cápita), y los municipios que figuraron entre los cinco primeros colocados en el Rank del IDSA están entre los más desarrollados económicamente en el estado. Además de que todos esos municipios presentaran un ICA superior a 0.5.

Cuadro 5. Variables utilizadas en el cálculo del IDSA y sus respectivos pesos

\begin{tabular}{ll}
\hline \multicolumn{1}{c}{ Indicador } & Pesos \\
\hline ICA & 0,1187 \\
Esperanza de vida al nacer & 0,3283 \\
Renta per cápita & 0,5346 \\
Tasa bruta de frecuencia escolar & 0,0098 \\
\hline
\end{tabular}

Origen: Resultados de la investigación. 
Por otro lado, los municipios de peores IDSA (América Dourada, Santa Brígida, Quijingue, Nordestina, Cansanção), con excepción del Indicador de Tasa Escolar, presentaron los demás indicadores inferiores al observado en la media del estado, destacándose nuevamente los indicadores socioeconómicos, en los que estos municipios presentaron una media $40 \%$ inferior a la estadual.

Según la clasificación de la ONU (2000), casi todos los municipios bahianos están agrupados como medio de desarrollo humano. En esta clasificación, el único que presentó IDH elevado fue el municipio de Salvador $(0,805)$.

Sin embargo, cuando se suman las condiciones de medio ambiente al cálculo del IDH, se tiene una reducción considerable de los índices de desarrollo. Con el IDSA, 93.7\% de los municipios bahianos fueron clasificados en la faja de bajo nivel (Gráfica 4). Este resultado puede ser explicado por la baja condición de vida, principalmente de saneamiento básico, en los municipios bahianos.

Se observa que la inclusión del ICA se constituye en un reductor en el nivel de desarrollo humano. A pesar de la reducción de la media general en el IDSA, fue posible verificar que en su mayoría los municipios de mayores IDH también presentaron los mayores IDSA.

Gráfica 4. Índice de Desarrollo Socioeconómico Ambiental e Índice de Desarrollo Humano de los municipios bahianos para el año de 2000

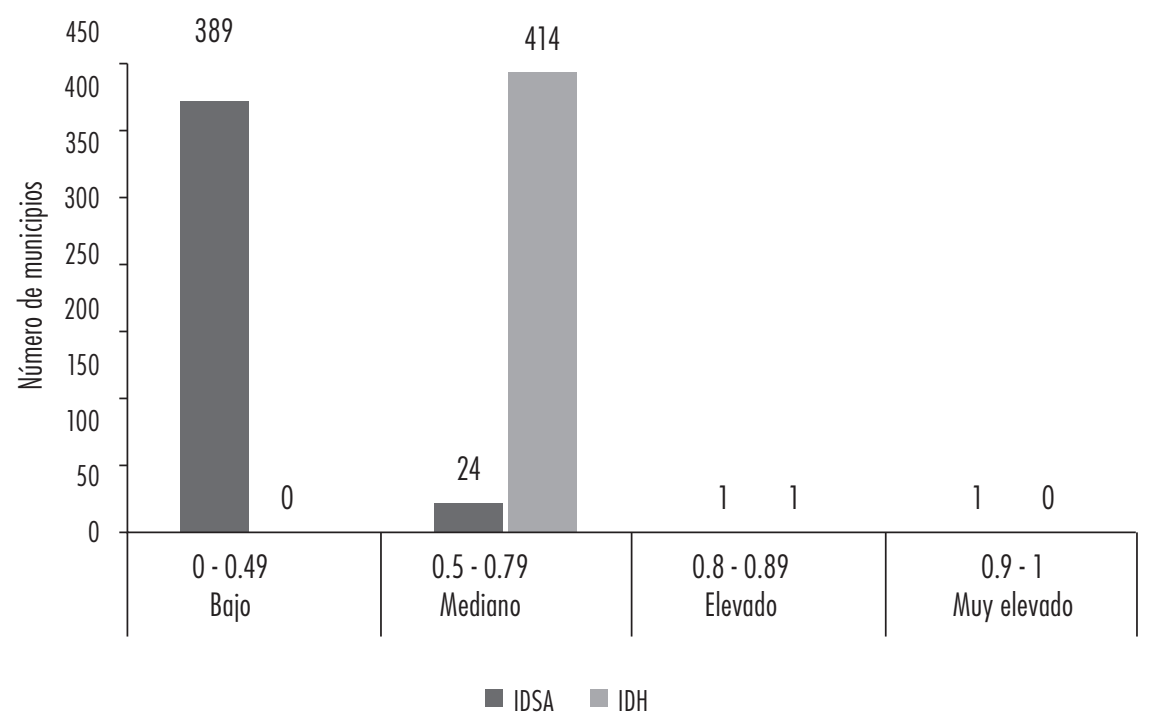

Origen: Resultados de la investigación. 
Por la Gráfica 5 se tiene la visualización de la distribución geográfica del IDSA. Vistos los bajos niveles de condiciones ambientales junto a la baja renta per cápita en el estado, casi la totalidad de los municipios bahianos son clasificados como de bajo nivel de Desarrollo Socioeconómico Ambiental.

Los cuatro mejores municipios bahianos clasificados en el IDH también fueron clasificados por el IDSA: Salvador, Lauro de Freitas, Itabuna y Madre de Deus. Los dos primeros poseen un buen rendimiento para el indicador de renta. Ya para los municipios de Itabuna y Madre de Deus, el ICA y la Esperanza de Vida al nacer explican su sustentación en el tercer y cuarto lugar, respectivamente. No obstante, el municipio de Feira de Santana fue superado por Paulo Afonso, justamente por presentar este último un ICA más elevado (Cuadro 6).

El cambio ocurrido en las últimas posiciones se dio principalmente por los pesos de los indicadores de renta per cápita y condiciones ambientales, en que los municipios con mayores indicadores lograron mejorar su posición en relación a los demás.

Gráfica 5. Disposición geográfica del IDSA en el Estado de Bahía.

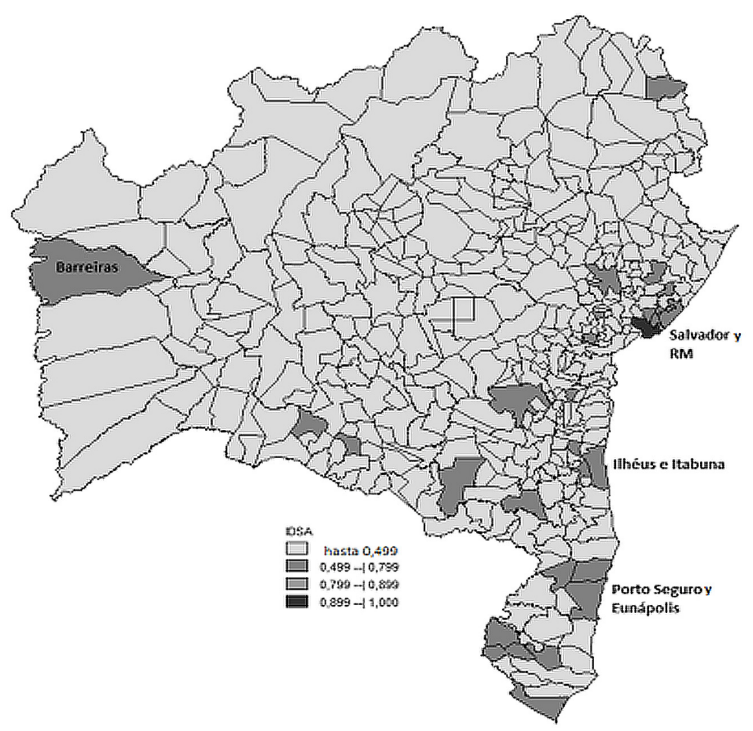

Origen: Resultados de la investigación. 
Cuadro 6. Los cinco municipios del Estado de Bahía de mejor y peor IDH e IDSA para el año de 2000

\begin{tabular}{lll}
\hline Posición & IDH & IDSA \\
\hline $1^{\circ}$ & Salvador & Salvador \\
$2^{\circ}$ & Lauro de Freitas & Lauro de Freitas \\
$3^{\circ}$ & Itabuna & Itabuna \\
$4^{\circ}$ & Madre de Deus & Madre de Deus \\
$5^{\circ}$ & Feira de Santana & Paulo Afonso \\
\hline $411^{\circ}$ & Monte Santo & América Dourada \\
$412^{\circ}$ & Santa Brígida & Santa Brígida \\
$413^{\circ}$ & Coronel João Sá & Quiïngue \\
$414^{\circ}$ & Quijingue & Nordestina \\
$415^{\circ}$ & Itapicuru & Cansanção \\
\hline
\end{tabular}

Origen: Resultados de la investigación.

Con la construcción del IDSA fue posible verificar una reducción en el índice medio de desarrollo del estado. Este hecho demuestra la importancia de la inclusión de variables ambientales en la cuenta de medida de desarrollo, principalmente para unidades de federación como Bahía en que el medio ambiente tuvo un peso de aproximadamente $12 \%$ en la determinación del índice. Según el IBGE (2010), en el periodo 2002-2008 Bahía fue uno de los estados brasileros que presentó, en términos relativos, mayor área desmatada (6.12\%), perdiendo apenas con Maranhão con 6.99\%. Es importante resaltar que la mayor parte de la leña en Brasil es carbón vegetal, utilizado inclusive en la siderurgia, obtenido de derrumbadas y quemas de vegetación nativa. En Bahía, los cerrados son los propósitos principales.

Otro gran problema del estado es la disposición de servicios básicos como abastecimiento de agua, recolección de basura y alcantarilla que todavía son muy precarios. Para tener una idea; del total de habitantes que viven en domicilios particulares permanentes de la zona urbana, apenas $62.2 \%$ posee alcantarilla sanitaria adecuada (red colectora); $2.3 \%$ de las habitaciones todavía no poseen ningún tipo de alcantarilla sanitaria, con pésimas condiciones para las poblaciones más pobres (IBGE, 2010). Éste es un escenario extremadamente negativo que fue generado por la creciente urbanización de las regiones metropolitanas, principalmente la de Salvador.

Las políticas ambientales de manera general tienden a concentrarse en la defensa de la biodiversidad. Las iniciativas dirigidas al uso racional de los recursos 
naturales y al límite de la capacidad del ecosistema son todavía pequeñas, tanto en calidad como en cantidad. Algunas iniciativas como el Programa de Saneamiento Ambiental de Bahía de Todos os Santos (Programa Bahía Azul) ${ }^{8}$ tuvo resultados satisfactorios para Salvador, pero los datos del interior del estado muestran los efectos de un ambiente sin saneamiento básico y sin condiciones socioeconómicas para asegurar un mínimo de calidad de vida.

\section{CONSIDERACIONES FINALES}

Este trabajo quiso identificar la asociación entre las condiciones socioeconómicas y las condiciones ambientales de los municipios bahianos, destacándose aspectos relacionados con el nivel de renta, el nivel de los recursos naturales, las condiciones de salud humana y la infraestructura sanitaria, principalmente. Se buscó incluso cuantificar el nivel de calidad ambiental de los municipios y construir un índice alternativo al IDH que ha considerado, además de componentes de naturaleza económica y social, componentes de naturaleza ambiental.

Los resultados revelaron que el Estado de Bahía posee un ICA medio de 0.34, o sea abajo del nivel de las condiciones ambientales del estado. Es importante resaltar que $80.24 \%$ de los municipios bahianos obtuvieron valores para el ICA inferiores a 0.50 y apenas dos municipios obtuvieron valores más grandes que 0.80 . El bajo nivel de preservación de las florestas y la precariedad del acceso a los servicios de saneamiento básico fueron los principales indicadores que caracterizaron los municipios con bajo nivel de ICA.

Vale destacar que 82 municipios que presentaron ICA superior a 0.5 , a pesar de poseer un padrón de infra estructura urbana relativamente buena, no están dando un destino adecuado a los residuos generados por la población. El no recogimiento de basura agrava las condiciones ambientales, ya que se ha visto que cuando no es recogida, es quemada (lo que afecta de manera negativa a la calidad del aire), enterrada (afecta de manera negativa a la calidad del suelo y de las aguas manantiales) o echada a los ríos (afecta de manera negativa los cursos de agua), afectando de manera negativa a las condiciones de vida de la población.

El Índice de Desarrollo Socioeconómico Ambiental construido presentó una media de 0.28 , lo que permitió concluir que las condiciones ambientales de los

8 Proyecto de intervención ambiental, centrado en alcantarillado sanitario, ampliación de la red de abastecimiento de agua y mejoría del sistema de recolección de basura. 
municipios estudiados constituyeron un factor reductor del nivel de desenvolvimiento humano, ya que el IDH tradicional clasifica la mayoría de los municipios bahianos en el intervalo de 0.5 a 0.79 .

Es importante reconocer que la calidad de vida está relacionada con las condiciones ambientales. De esta manera, mejoras ambientales pueden dar como resultado avances en las condiciones económicas y sociales (impactos sobre la pobreza). El reciclaje de residuos, la conservación del agua y de la energía son ejemplos de cómo crear empleos y mejorar la calidad de vida de la población. El Estado de Bahía está todavía muy abajo en términos de iniciativas de este tipo.

Es importante que las autoridades gubernamentales estén atentas en reconocer la importancia de proveer servicios básicos adecuados, principalmente los de saneamiento básico. La importancia de la adopción de medidas destinadas a mejorar la infraestructura sanitaria, de salud, de habitación, de áreas de preservación, de ocupación del espacio físico, etcétera, como la necesidad de caminar en dirección al desarrollo de manera sustentable, se vuelven cuestiones fundamentales en la actualidad. El resultado de acciones con este objetivo llevaría a un avance en la calidad de vida y en la calidad ambiental de las comunidades beneficiadas, generando un efecto positivo para el país como un todo.

\section{BIBLIOGRAFÍA}

Barbier, E., The Economic Linkages Between Rural Poverty and Land Degradation: Some Evidence from Africa, Agriculture, Ecosystems and Environment, 82, 2000, pp. 355-370.

Bojö, J., J. Bucknall, K. Hamilton, N. Kishor, C. Kraus, P. Pillai, Environment Chapter, Poverty Reduction Strategy Papers'Source Book, Washington, DC, World Bank, 2001.

Braga, T. M. B.; Freitas, A.P.G.; Duarte, G.S.; Carepa-Souza, Julio. Índices de Sustentabilidade Municipal: O Desafio de Mensurar. 2003. Disponible en: http://www.cedeplar.ufmg.br/pesquisas/td/TD\%20225.pdf. Acceso el 15 de diciembre de 2010.

Corrar, L. J., E. Paulo, J. M. Dias Filho, Análise Multivariada, Fipecafi, São Paulo, Atlas, 2007.

DSD (Division for Sustainable Development), United Nations, Departament of Economic and Social Affairs. Disponible en: <www.un.org/esa/dsd/dsd_ aofw_ind/ind_index.shtml>. Acesso em: 4 jul. 2009.

Ekbom, A., J. Bojö, Poverty and Environment: Evidence of Links and Integration into the Country Assistance Strategy Process, 1999. Disponible en: <http:// 
www.staff.ncl.ac.uk/david.harvey/AEF806/WBPovEn.pdf> Acesso em 20 de out. de 2011.

Gallopín, G. C., "El ambiente urbano y la planificación ambiental”, en: Medio ambiente y urbanización, Buenos Aires, CLACSO/CIFCA, 1982.

IBge (Instituto Brasileiro de Geografia e Estatística), Censo Demográfico 2000. Disponible en: http://www.sidra.ibge.gov.br/bda/popul/default.asp?z=t\&o $=23 \& \mathrm{i}=\mathrm{P}$. Acesso em 20 mai 2009 .

, Censo Agropecuário 2006. Disponible en: http://www.sidra.ibge.gov .br/ $\mathrm{bda} /$ pesquisas/ca/default.asp?o=2\&i=P. Acesso em jul 2009. , Pesquisa de Informaçóes Básicas Municipais 2000. Disponible en: http:// www.sidra.ibge.gov.br. Acesso em jul 2009.

, Indicadores de Desenvolvimento Sustentável, Brasil 2010. Disponible en: http://www.ibge.gov.br/home/geociencias/recursosnaturais/ids/ids2010. pdf. Acesso em 20 de out. de 2011.

Leff, H., Saber Ambiental, 3a edição. Ed. Vozes. 2004.

Leite, M. De P., "Trabalho e sociedade em transformação", Sociologias [online], 2000, num. 4, pp. 66-87.

Parikh, J., 'Poverty -Environment- Development Nexus International", Journal of Global Environmental, Issues 2, 344-65, 2002.

Prescott-Allen, R. The Barometer of Sustainability, IUCN, 2005. Disponible en: $<$ http://www.iucn.org/themes/eval/english/barom.htm>. Acceso el $17 \mathrm{de} \mathrm{di-}$ ciembre de 2010.

Ross, J. L. S., Geografia do Brasil, $4^{a}$ ed., São Paulo, Editora da Universidade de São Paulo, 2001.

Sachs, Ignacy, Rumo à Ecossocioeconomia. Teoria e prática do desenvolvimento. Ed. Cortez, pp. 472, 2007.

SEI (Superintendência de Estudos Econômicos e Sociais da Bahia). Disponible en: http://www.sei.ba.gov.br/. Acesso em nov. 2009.

Sen, Amartya, Desenvolvimento como liberdade, São Paulo, Schwarcz, 2008, $409 \mathrm{p}$.

SEPlan (Secretaria do Planejamento da Bahia). Disponible en: www.seplan. ba.gov.br/arquivos/rel_atividades2005/html/pdf/vol.2/Riquezas/Riquezas. pdf. Acesso em: 01 mar. 2009.

undP (United Nations Development Programme). Disponible en: www.undp. org/. Acesso em: dez 2009. 